\title{
ASSESSING MOTORCYCLE CRASH-RELATED HEAD INJURIES USING FINITE ELEMENT SIMULATIONS
}

\author{
Toma, M. ; Njilie, F. E. A. ; Ghajari, M. ${ }^{* *}$ \& Galvanetto, U. \\ *Altair Development France; 2, rue de la Renaissance; Antony 921 60; France \\ ${ }^{* *}$ Department of Aeronautics; Imperial College London; SW7 2AZ; United Kingdom \\ E-Mail: fnjilie@europe.altair.com
}

\begin{abstract}
Motorcycle crash-related fatalities and injuries have a relatively increasing tendency compared to other vehicles. The new development of safety devices and technologies for prediction of their behaviour are therefore also increasingly important. Motorcycles have the least amount of protective devices amongst vehicles. A small disturbance in the motion of motorcycles can expose the riders to severe impacts leading to injuries especially in the appendicular part of the body, but the severest injury is usually to the head. Head injuries are the most common cause of death amongst motorcyclists (approximately $45 \%$ ). Thus, naturally, the main protective equipment preventing motorcyclists from fatal injuries is the helmet. In this study, detailed finite element models of helmet and human head are used to simulate and analyse the impacts on a protected and unprotected head in a scenario typical for motorcycle-related collisions.

(Received in February 2010, accepted in April 2010. This paper was with the authors 1 month for 1 revision.)
\end{abstract}

Key Words: FEA, Motorcycle Crash, Head Impact, Helmet, Design of Experiments

\section{INTRODUCTION}

There are three main factors determining the injuries; namely the mass of colliding vehicles, the change in velocity of vehicles during impact and the use of safety devices [1]. Motorcycle crash-related fatalities account for $16 \%$ of total road fatalities in Europe - every year there are approximately over 6000 powered two-wheeler (PTW) fatalities [2]. Although there are other means of protective devices already developed or still being developed for PTW riders [3-5], helmets are still almost the only items of equipment in use that prevent riders from fatal injuries to the head. The performance of the helmets in accidents is tested according to one of the accepted helmet testing standards, namely ECE 22.05 (EU-2000), BS 6658 (UK-1985), FMVSS 218 (USA-2003), Snell M2005 (USA-2005) and AS/NZS 1698 (2006). The aim of using computer simulations is to diminish the expense of conducting experimental tests, including the studies carried out to measure head impact. A study on the influence of mechanical characteristics of the brain tissue on head injury criteria using finite element analysis (FEA) shows that head impact criterion (HIC), a widely used head injury measure for dummies derived from the time history of an accelerometer mounted at the centre of gravity of a dummy's head, is insensitive to the type of material used to model the brain tissue [6], thus, in this sense, justifying the use of different models. Evaluations of head injuries using FEA have been performed in numerous studies [7-9], some of them using helmets too [10, 11]. The main difference in this study is the use of more detailed models of helmet and head and a focus on the motorcycle crash-related impact conditions in order to model more realistic scenarios, as well as a different approach to analysing the results. The improvement in computer technology has been phenomenal in recent decades allowing the use of more detailed models without unnecessary limitations to their geometry and complexity. 


\section{HELMET AND HEAD MODELS}

The helmet model used in this study has been developed and tested against three of the above mentioned standards, namely ECE 22.05, Snell M2005 and AS/NZS 1698, at Imperial College London [12]. New materials, potentially suitable for the improvement of the energy absorption levels in existing helmets, are currently being investigated there. The finite element model of the head used here is a part of HUMOS2 (HUman MOdel for Safety), a project funded by the European Commission representing a large range of the European population and allowing an accurate injury risk prediction for people involved in road accidents [13]. The head of this model is validated using the frontal and lateral head drop tests compared with HybridIII dummy model results and the head impact test compared with the results of Trosseille's test [14]. The helmet model consists of an outer shell, three foam liner components with different material properties and a chin strap, see Fig. 1 . The outer shell is a composite made of four layers with varying material properties [12]. The dimensions of the helmet correspond to headform size ' $\mathrm{O}$ ', the largest size according to the standards.
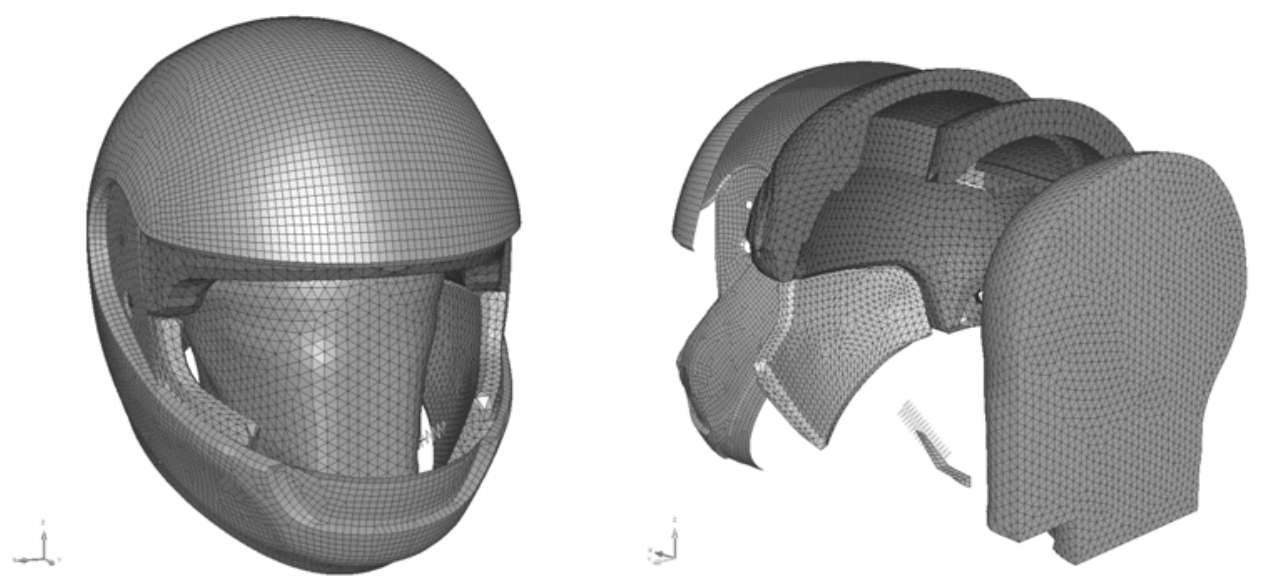

Figure 1: Structure of the Imperial College helmet model.
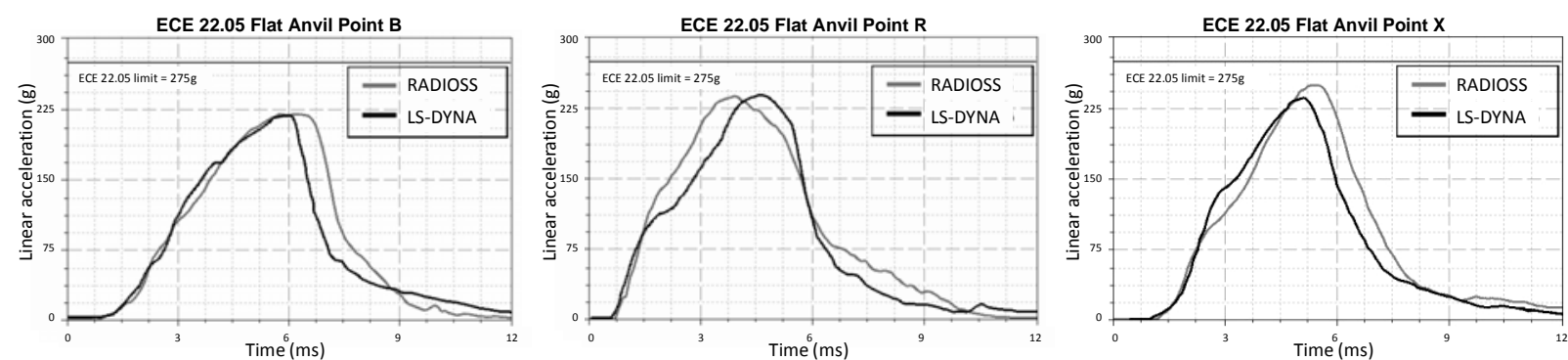

Figure 2: Results validating the translation of the helmet model from LS-DYNA to RADIOSS.

The helmet model from Imperial College is developed in LS-DYNA format [15]. However, the HUMOS2 model used in this study is created using RADIOSS. In order to couple these two models together, the helmet model is translated to RADIOSS format [16]. The translation is validated running several simulations in both formats, see Fig. 2. The plots show resulting linear accelerations versus time for impact against a flat anvil in several points of impact. Besides translating the helmet model to RADIOSS and consequently validating it, the coupling of HUMOS2 head with helmet model requires also careful attention when fitting the head into the foam liners surrounding it inside of the helmet, see Fig. 3. Some nodes of the foam liners have to be slightly morphed to adjust their geometry to the geometry of the head, 
as it would happen in real life too, when putting a helmet on the head. No pre-stress in the foam is defined; it is negligible in comparison to the stresses caused by the impact. Proper contact interfaces, with static Coulomb friction of 0.5 between the head and the interior parts of the helmet, are defined. The corresponding weights of head, neck and helmet are 4.84, 1.81 and $0.86 \mathrm{~kg}$, respectively. The total number of elements of the coupled model is 77264 consisting of 196 1D elements (spring elements), 16784 2D elements (triangular and quadrilateral shell elements) and 60284 3D elements (tetrahedral and hexahedral solid elements).
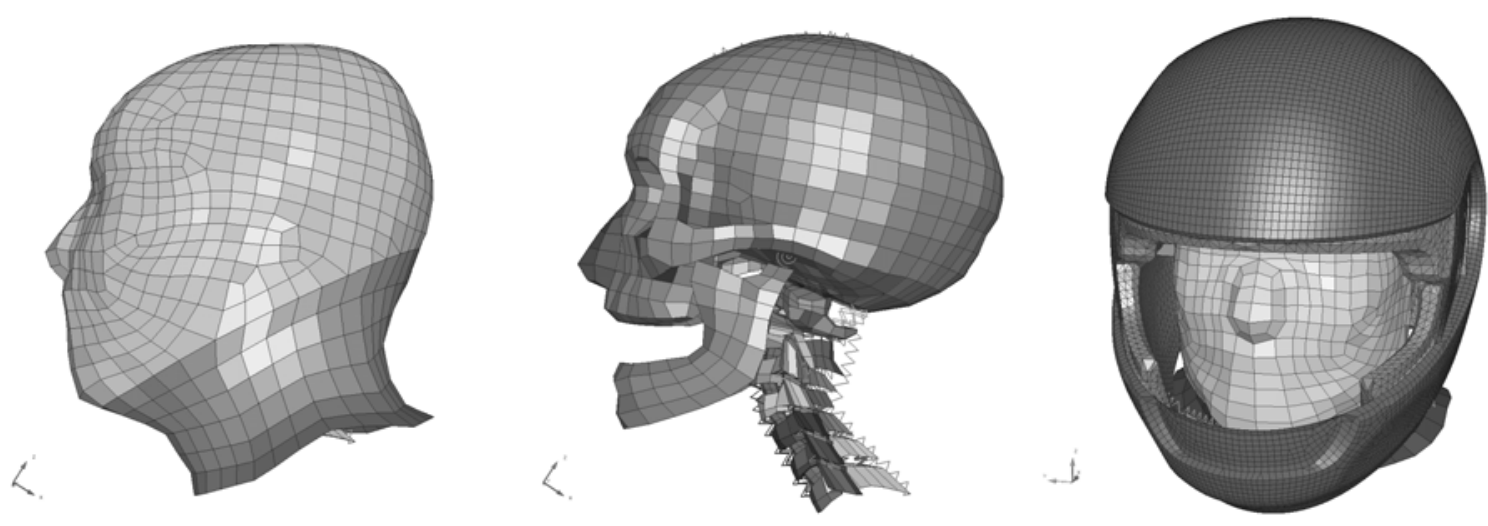

Figure 3: The HUMOS2 head with and without skin and flesh and coupled with helmet model, respectively.

\section{IMPACT CONDITIONS}

ISO13232 specifies seven basic impact configurations recommended for evaluating rider protective devices. One of them is used in this study to model a motorcycle-car collision using simplified (no engine) two-wheeler vehicle and simplified (semi-rigid) HYBRIDIII model as a rider dummy model, see Fig. 4. The corresponding weights of the two-wheeler vehicle, dummy and the other vehicle are $8.23,63.68$ and $1711.5 \mathrm{~kg}$, respectively. These simplified models are chosen because this simulation is used here only for the sake of completeness in order to demonstrate the entire process of a numerical simulation of motorcycle-car collision from its kinematics to the injuries assessment. The main objective of this study is to evaluate the head injuries from head impact in relation to motorcycle-related crashes. Numerous studies have been performed on numerical simulations of motorcycle-car collisions elsewhere [5, 17-19]. The impact configuration code chosen from the ISO13232, in order to extract the impact conditions between the dummy and the car, is 413-0/13.4. The first part of the code consists of three digits describing the car contact point, the motorcycle contact point and the relative heading angle, respectively, followed by a dash and the car impact speed and the motorcycle impact speed, respectively, in $\mathrm{m} / \mathrm{s}$. The contact points for this code are shown in Fig. 4. The car is not moving, $0 \mathrm{~km} / \mathrm{h}$, and the motorcycle has an impact speed of $48.24 \mathrm{~km} / \mathrm{h}$ (29.97 mph), i.e. $13.4 \mathrm{~m} / \mathrm{s}$ according to the configuration code. This configuration is modelled using HyperCrash and computed using RADIOSS, obtaining the resulting position of the contact point between the dummy and the car. The related impact speed is extracted at this point too.

Fig. 5 represents the resulting position between the dummy and the car at the moment of impact between them. The head is the first relevant body part in contact with the car, namely the upper part of the window of the side door. The speed of the head perpendicular to the window at the moment of contact is $46.01 \mathrm{~km} / \mathrm{h}(28.59 \mathrm{mph})$. 


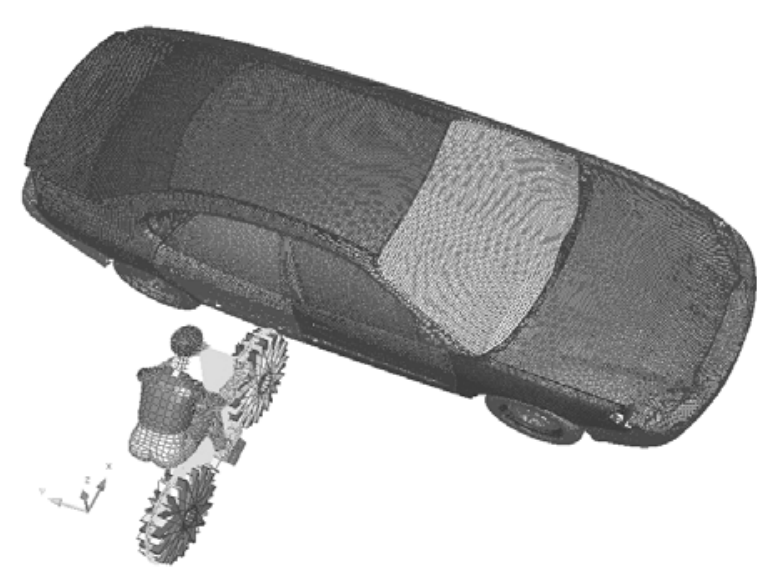

Figure 4: Motorcycle-car impact configuration.

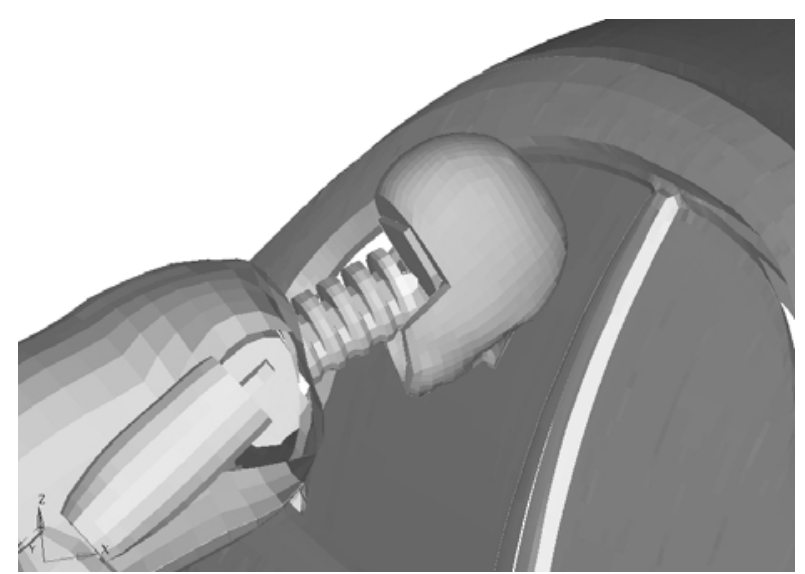

Figure 5: The position of the dummy in contact with the car.

\section{INJURY ASSESSMENT}

It is common practice, in the injury assessment procedure, to evaluate the level of injuries using only the part of the body which is to be affected by the impact. The interest here is focused on the short time period of the impact only and the influence of the remaining mass is already incorporated into the impact speed extracted from the simulation using the full dummy model. Therefore, no added mass or inertia is included. Similarly, only the part of the car in contact with the head is considered during the next step of the procedure. The boundary conditions for the window are obvious; the points on its boundaries are fixed in all directions, as though the glass is fixed in the surrounding structure of the car. The HICs for the simulations in cases of the head with and without the helmet are 732 and 12958, respectively. A value of 1000 corresponds to $18 \%$ probability of a severe head injury, a $55 \%$ probability of a serious injury and a $90 \%$ probability of a moderate head injury to the average adult [20]. Fig. 6 shows both the models with and without the helmet and the distribution of the energy transmitted during the impact at the same time instant after the fist contact occurs. As expected, the figure shows that the skull and brain absorb less energy with the helmet protection on, as most of it is absorbed by the helmet itself.

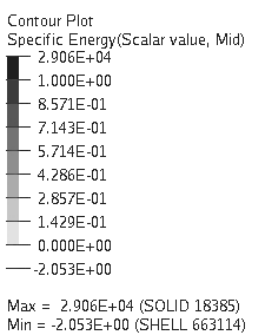

Max $=2.906 E+04$ (SOLID 18385)
Min $=-2.053 E+00($ SHELL 663114)

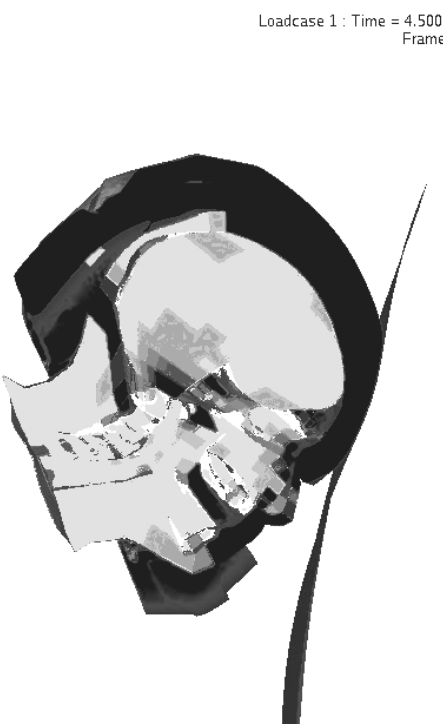

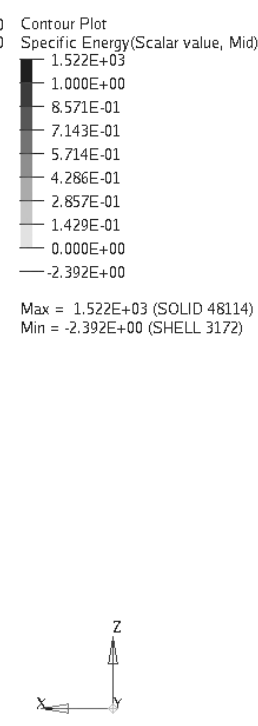

Loadcase $1:$ Time $=4.500000$
Frame 10

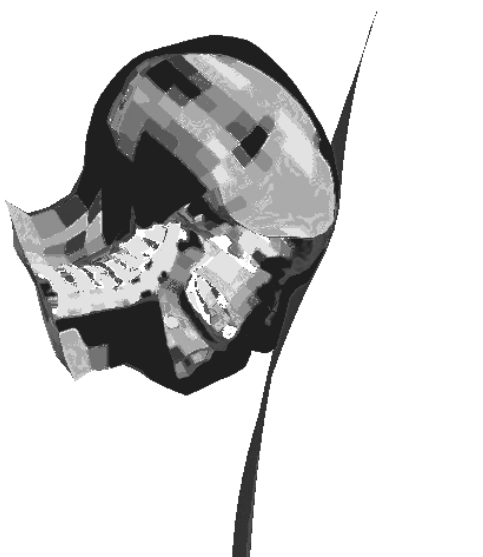

Figure 6: Specific energy in the models with and without the helmet, respectively. 


\section{DESIGN OF EXPERIMENTS}

The survival of a motorcyclist can depend on the choice of materials in the helmet and/or the window. Therefore, the question arises about the effect of the used materials on the object of interest, i.e. for the present study the HIC and the maximum value of the internal energy within the brain. Design of Experiments (DoE) can be performed in order to evaluate the factors that control the HIC or other objectives. A set of control tests is conducted, and these are analysed, in order to determine the relationship between the materials affecting the process and the output of that process. Three variables are chosen to perform the DoE, namely the scale factors for yield curves of two of the foams surrounding the head and the Young's modulus of the window. In other words, all these variables determine the stiffness of the chosen materials. Since the impact is to the frontal bone of the skull, the third foam, protecting the cheeks, is not considered for the DoE.

Table I: Variables determining the stiffness of the chosen materials and the corresponding HICs.

\begin{tabular}{|c|c|c|c|c|c|}
\hline Run & Foam Liner 1 & Foam Liner 2 & Window (MPa) & HIC & Energy in Brain (mJ) \\
\hline 1 & 58 & 61 & 68000 & 491 & 105 \\
\hline 2 & 58 & 61 & 72000 & 532 & 108 \\
\hline 3 & 58 & 63 & 68000 & 1829 & 106 \\
\hline 4 & 58 & 63 & 72000 & 996 & 110 \\
\hline 5 & 60 & 61 & 68000 & 476 & 129 \\
\hline 6 & 60 & 61 & 72000 & 671 & 134 \\
\hline 7 & 60 & 63 & 68000 & 654 & 131 \\
\hline 8 & 60 & 63 & 72000 & 1006 & 136 \\
\hline
\end{tabular}
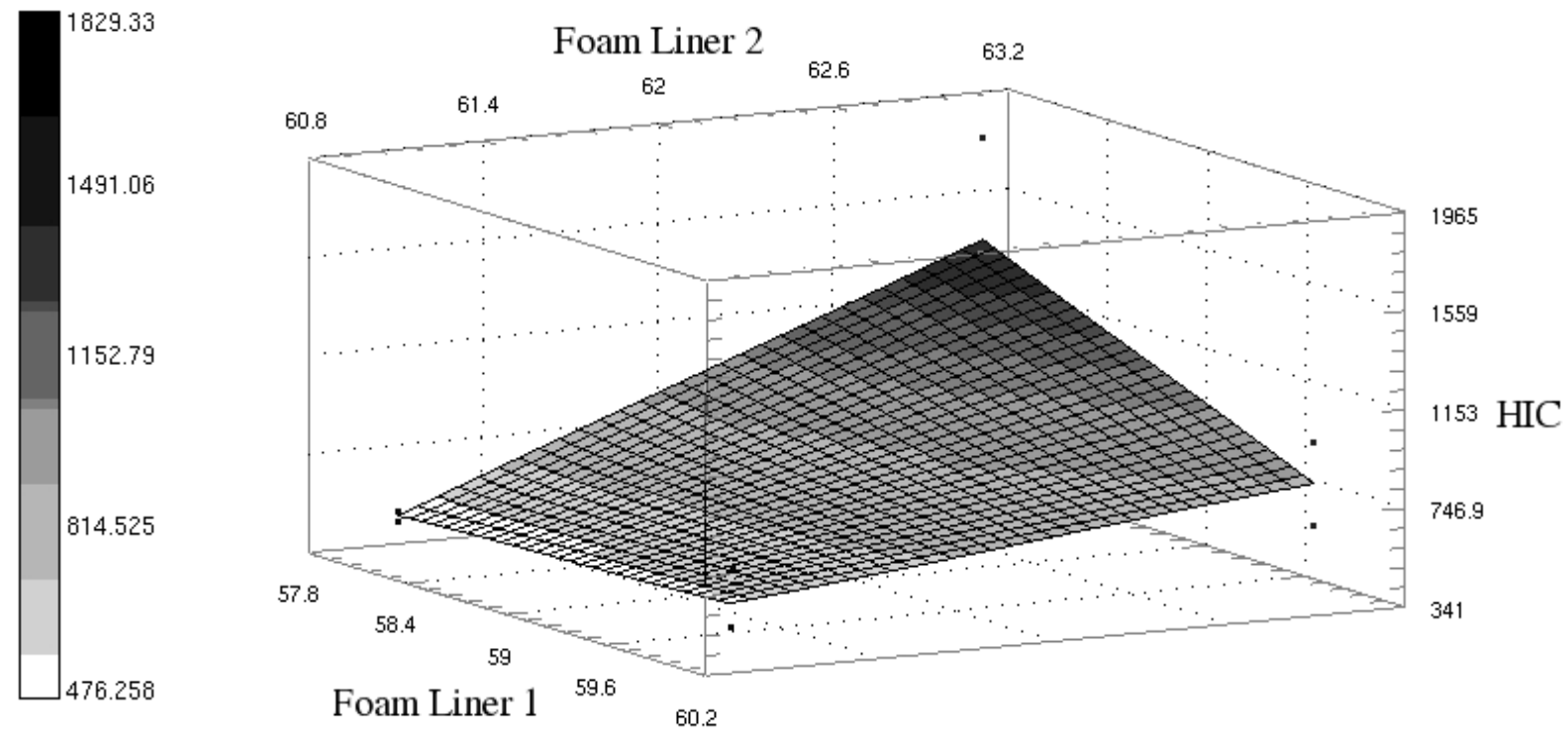

Figure 7: Interpolated response surface.

The values for all the materials are summarized in Table I, where foam liner 1 represents the largest liner surrounding most of the skull and foam liner 2 represents the smaller one on top of the head, see Fig.1. Each of the variables has two different values and altogether they complete eight different combinations, i.e. full factorial DoE measuring the response of every possible combination of factors. Thus, eight simulations of these combinations are computed using RADIOSS solver and analysed using HyperStudyDSS. The solutions are summarized in 
the last two columns of Table I. The values of corresponding HICs vary substantially around the mean value of 832. Therefore, naturally, the values of stiffness of the chosen materials could predetermine the survival of the motorcycle rider in the given scenario. Fig. 7 depicts the effect of the stiffnesses of both of the liner components on the HIC. The higher the stiffness of liner 2, the higher the HIC. However, it is not as clear with the stiffness of liner 1. In case of high stiffness of liner 2, the increasing stiffness of liner 1 decreases the chance of head injury. In case of low stiffness of liner 2, the increasing stiffness of liner 1 has only a small influence on the HIC. An explanation of this combined effect is not really straightforward. Possibly due to the geometry of both of the liners, having liner 2 stiffer than liner 1 causes an additional movement inside the helmet which influences the global deceleration of the head during impact.

Fig. 8 shows the effects and the combined effects of all three stiffnesses on the HIC. Every graph has three horizontal dot-dashed lines. The central one represents the mean value, 832. The three graphs on the diagonal show the individual effects of all three variables separately. The stiffness of the foam liner 2 has the greatest variation around the mean value. Similarly as observed from Fig. 7, with an increasing value of the stiffness of foam liner 2, the HIC increases. It is the opposite in the case of foam liner 1 , with a smaller effect on the HIC. The stiffness of the window has only a minimal effect on the HIC. The other graphs show the combined effects. The $+1(-1)$ in those graphs means that the corresponding dashed line in the graph represents the effect of the stiffness of the material stated at bottom of the figure when the material stated at the left has its maximum (minimum) value. For example, the first graph in the second row shows that with increasing stiffness of foam liner 1, and maximum value of the stiffness of liner 2, the HIC decreases; while in case of a minimum value of the stiffness for liner 2 the HIC increases only slightly, as also previously concluded from Fig. 7.
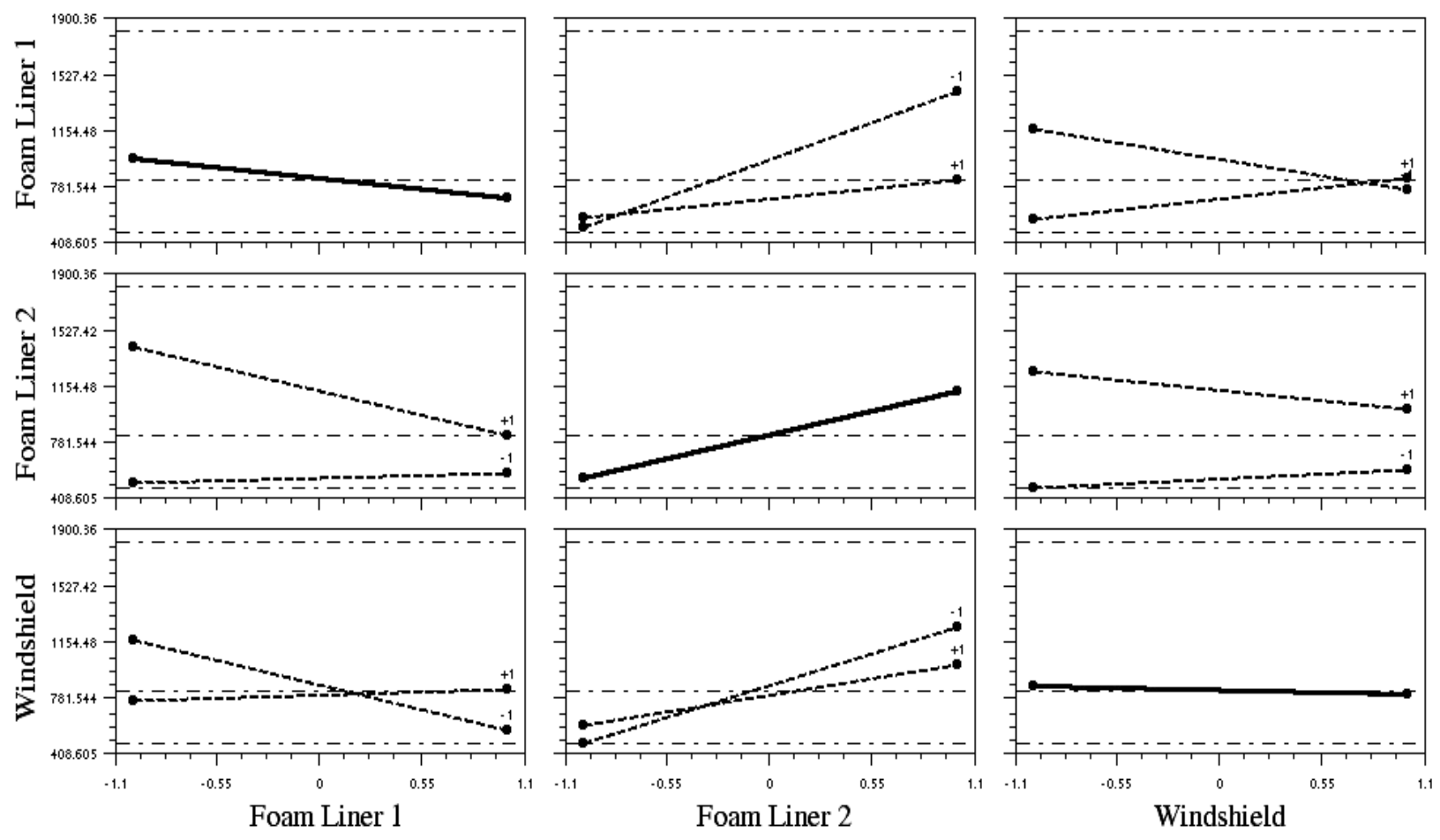

Figure 8: The effects and combined effects on HIC.

Similarly, since a detailed head model is used, other objectives concerning the head model can be evaluated within the scope of a DoE. Fig. 9 shows the individual and combined effects of the variables on the maximum value of the internal energy absorbed by the brain during the 
impact. The combined effects are linear, and it is therefore rather straightforward to analyse the outcome. The foam liner 1 has the highest effect on the energy absorbed within the brain, and, considering the point of impact, the foam liner 2 has the smallest effect. The stiffness of the window, in comparison to the stiffness of the foam liner 1, influences the internal energy of the brain only slightly. Essentially, there are two levels of the internal energy, lower level for lower stiffness of the foam liner 1 and higher level for the higher stiffness. Interestingly, the run number 5, see Table I, gives the smallest HIC, but, at the same time, a higher level of the internal energy. Although there are no documented threshold values for the HUMOS2 head model to correlate the energy absorbed by the brain and the level of head injuries, the fact that higher levels of the internal energy can occur together with a low value of HIC suggests that the HIC alone, at least in cases of direct impact to the head protected by a more detailed helmet, might not be enough to accurately predict the level of head injuries.
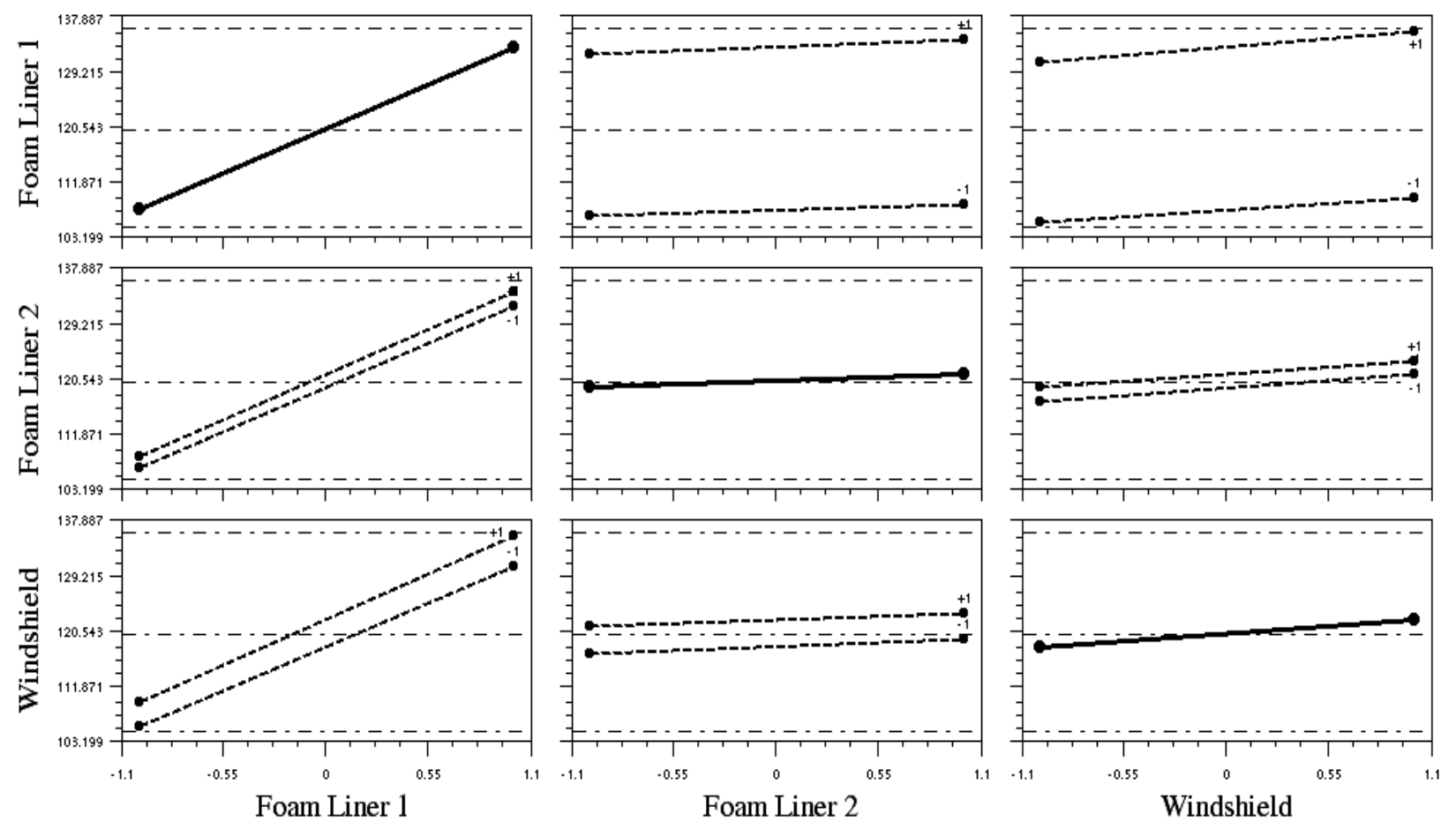

Figure 9: The effects and combined effects on internal energy in the brain.

\section{CONCLUSION}

The entire process of the computational modelling of an accident scenario from the first impact between the PTW vehicle and the other vehicle up to the contact of the PTW rider with the other vehicle, including the consequent injury assessment, is presented. Thus, the injury assessment procedure can be summarized in three steps. Firstly the kinematic run is executed with the impact conditions between the two vehicles; secondly the impact conditions between the PTW rider and the other vehicle are extracted at the point of their impact, namely the impact speed and the direction of impact of the relevant body part with respect to the car, and thirdly another run is executed involving the relevant body and car parts with the appropriate boundary and impact conditions. The difference between protected and unprotected head impacts is shown. The DoE analysis is performed on the complex helmet model coupled with a detailed human head model. As the development of the protection evolves, the protective equipments are becoming more complex. It is concluded that the DoE can be very useful in analysing the combined effects of various parts of the protective 
equipment used. As shown, the resulting HIC can depend on interaction between parts of the helmet. A change to the property of one part can cause a decrease or increase of HIC depending on a property of another part of the helmet. A combination of material properties giving low HIC and higher level of internal energy within the brain is found, suggesting that the sled-test-based HIC might not in itself be accurate enough for injury prediction in a case of direct impact of a helmeted head.

\section{ACKNOWLEDGMENTS}

The work presented here is a part of the research network MYMOSA (MotorcYcle and MOtorcyclist SAfety) financed by the $6^{\text {th }}$ Framework Program (Marie Curie Actions) of the European Union, under the contract MRTN-CT-2006-035965. Also, thanks goes to Douglas Robertson for proofreading in English.

\section{REFERENCES}

[1] Broyles, R. W.; Narine, L.; Clarke, S. R.; Baker, D. R. (2003). Factors associated with the likelihood of injury resulting from collisions between four-wheel drive vehicles and passenger cars, Journal of Accident Analysis and Prevention, Vol. 35, 677-681

[2] European Communities (2001). COST 327, Motorcycle Safety Helmets, Final Report of the Action, Belgium

[3] European Communities (2004). APROSYS (Advanced Protection Systems), supported by the $6^{\text {th }}$ Framework Program (Marie Curie Actions) of the European Union, under contract number TIP3CT-2004-506503

[4] Namiki, H.; Nakamura, T.; Iijima, S. (2005). A Computer Simulation for Motorcycle Rider Injury Evaluation in Collision, Honda R\&D Technical Review, Vol. 17, No. 2, 153-160

[5] Chawla, A.; Mukherjee, S. (2007). Motorcycle safety device investigation: A case study on airbags, Sadhana, Vol. 32, No. 4, 427-443

[6] Aida, T. (2000). Study of human head impact: brain tissue constitutive models, Ph.D. thesis, Department of Mechanical Engineering, West Virginia University

[7] Brands, D. W. A. (2002). Predicting brain mechanics during closed head impacts, University Press Facilities, Eindhoven

[8] Afshari, A.; Rajaai, S. M. (2008). Finite element simulations investigating the role of the helmet in reducing head injuries, International Journal of Simulation Modelling, Vol. 7, No. 1, 42-51

[9] Klein, S. (2000). Finite element modelling of human head, Ph.D. thesis, Department of Aeronautics, Royal Institute of Technology, Sweden

[10] Kostopulos, V.; Markopoulos, Y. P.; Giannopoulos, G.; Vlachos, D. E. (2002). Finite element analysis of impact damage response of composite motorcycle safety helmet, Composites Part B: Engineering, Vol. 33, 99-107

[11] Deck, C.; Willinger, R. (2006). Multi-directional optimisation against biomechanical criteria of a head-helmet coupling, International Journal of Crashworthiness, Vol. 11, 561-572

[12] Ghajari, M.; Gaetano, D. C.; Galvanetto, U. (2008). Deliverable no. 3.1, MYMOSA, Sixth Framework Programme Marie Curie Research Training Networks, European Commission, WP31_310308.doc, 37p

[13] HUMOS2. Project funded by the European Commission under the Competitive and Sustainable Growth Programme, Contract G3RD-CT-2002-00803, from http://humos2.inrets.fr, accessed on 04-04-2010

[14] Arnoux, P. J.; Thollon, L.; Kayvantash, K. (2001). The RADIOSS Human Model for Safety (HUMOS), Validation of the Radioss HUMOS model, Definition of a model evaluation procedure, Altair Development France, 51p

[15] Hallquist, J. O. (2007). Ls-Dyna theory manual, Livermore Software Technology Corporation

[16] Toma, M. (2008). Conversion of helmet LS-DYNA model prepared by MYMOSA-WP3, Altair RADIOSS Safety Models, Internal Report Version 1.0, Altair Development France, Antony, 


\section{France}

[17] Chawla, A.; Mukherjee, S.; Mohan, D.; Bose, D.; Rawat, P.; Sakurai, M.; Nakatani, T. (2004). FE simulations of motorcycle-car frontal crashes, validation and observations, JARI Research Journal, Vol. 26, No. 10: 587-593

[18] Deguchi, M. (2005). Simulation of motorcycle-car collision, $19^{\text {th }}$ International Technical Conference on the Enhanced Safety of Vehicles, Washington D.C.

[19] Mukherjee, S.; Chawla, A.; Mohan, D.; Singh, M.; Sakurai, M.; Tamura, Y. (2001). Motorcyclecar side impact simulation, IRCOBI Proceedings, 133-141

[20] Mackay, M. (2007). The increasing importance of the biomechanics of impact trauma, Sadhana, Vol. 32, No. 4, 397-408 